\title{
Possible Skin Fragment Evidence is Found in Incomplete Fingerprints
}

\author{
A. Villena, ${ }^{*}$ A. Calabro, ${ }^{* *}$ D. Becker, ${ }^{* *}$ J.Morales*** \\ * Bergen County Technical High School, Law and Justice Department, 504 Rt. 46 West, Teterboro, \\ NJ 07608 \\ ** Bergen County Academies, Microscopy Laboratory, 200 Hackensack Avenue, Hackensack, NJ \\ 07601 \\ *** City College of New York, Electron Microscopy Facility, 160 Convent Avenue, New York, NY \\ 10031
}

Latent fingerprints are chance or accidental impressions left by friction of skin ridges on a given surface. Incomplete prints may be in inadmissible as evidence, limiting their usefulness in forensic science. We have conducted observations by scanning electron microscopy to determine if skin fragments may be found in incomplete fingerprints. Such skin fragments may be used as a source of DNA for genetic identification.

Prior to making a fingerprint, hands were washed and dried off with a paper towel. The substrate (aluminum or glass slide) was cleaned with alcohol, dried with Kimwipes and dusted off with pressurized gas (DustOff). The right thumb was then pressed against a $25 \mathrm{~mm}$ aluminum SEM stub or a glass slide. Prints made on aluminum pins were observed without any coating at $2 \mathrm{kV}$ in high vacuum mode. Prints made on glass slides were carbon coated and observed in high vacuum mode at $30 \mathrm{kV}$. An FEI Quanta 200 3D SEM was used to obtain secondary electron micrographs. As a control, small pieces $(1 \mathrm{~mm})$ of skin were mounted on SEM pins with carbon tape and observed, uncoated at $30 \mathrm{kV}$.

Observations made of prints show the presence of oil droplets along the skin ridge imprint (Fig. 1AD) and the presence of particles (Fig. 1C-E) along the ridges. These particles (Fig. 1C-E) are similar in morphology to control skin fragments (Fig. 1F and G). The flattened morphology of the particles found in prints (Fig. 2C-E) is consistent with the flattened morphology of squamous epithelial cells known to be present on the skin surface (Fig. 1F-G). Although, charging could have been eliminated by working in low vacuum mode, we found that charging was helpful in making the particles or skin fragments more identifiable. Some of the skin fragments found in prints were as long as 67 um (Fig 2E). Similar results were obtained from prints made on glass slides (results not shown).

Fingerprints have already been used as potential sources of DNA for genetic identification (Balogh et al, 2003). The collection of microscopic skin fragments from fingerprints followed by DNA fingerprinting could help law enforcement and forensic scientists link a particular suspect to a crime, even if the print is incomplete and/or smudged.

Cited Reference:

M.K. Galogh, J. Burger, K.Bender, P.M. Schneider, K.W. Alt, 2003. STR genotyping and mtDNA sequencing of latent fingerprint on paper. Forensic Science International 136:188-195. 

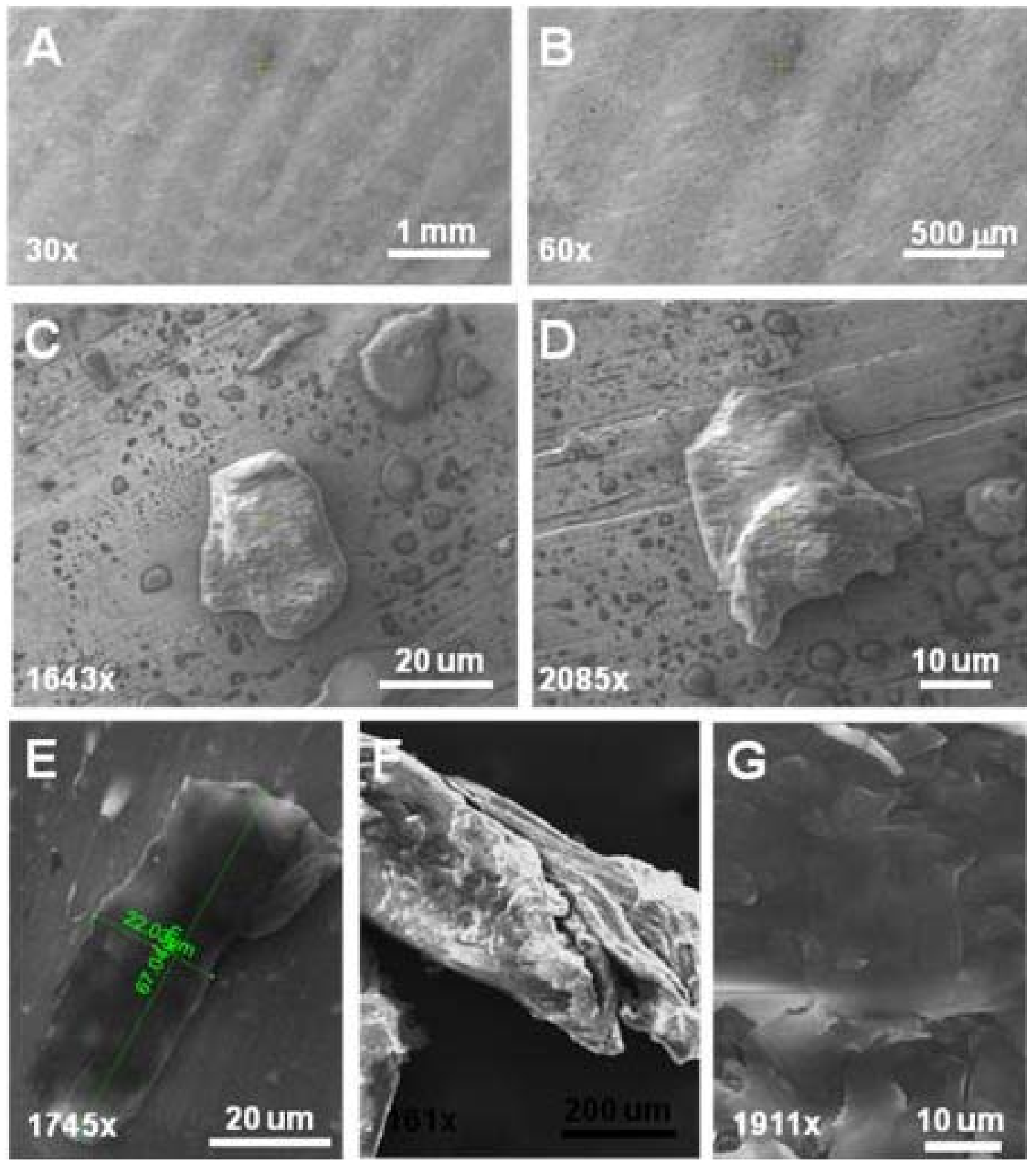

FIG. 1. Secondary electron micrographs of a fingerprint (A-E) and a skin fragment (F-G). Panels A and $\mathrm{B}$ are low magnification images of a partial fingerprint, notice the impressions made by skin ridges. At higher magnifications fragments of skin may be observed along the impressions made by skin ridges (C-E). All specimens were observed in high vacuum mode without metal or carbon coating. Charging is a desirable artifact in this case since it helps locate skin fragments along the ridges. 